\title{
Relações entre precipitações intensas de diferentes durações para desagregação da chuva diária em Santa Catarina
}

\author{
Álvaro J. Backㄹ, José L. R. O liveira² \& Alan Henn²
}

\section{RESU MO}

Com este trabalho objetivou-se determinar as relações entre precipitações de diferentes durações para 0 Estado de Santa Catarina. Foram estabelecidas as séries de máximas anuais de chuva com duração de 5 a 1440 min de treze estações pluviográficas de Santa Catarina. Para cada duração foram estimadas as precipitações máximas com período de retorno de 2 a 100 anos, com base na distribuição de $\mathrm{G}$ umbelChow. A relação média entre a chuva de $24 \mathrm{~h}$ e a chuva de um dia independe do período de retorno, com média para o estado de 1,17. Ao se analisar o horário da leitura da precipitação diária verificou-se que esta relação varia de 1,12 a 1,20 . Em referência à chuva de $1 \mathrm{~h}$ em relação à chuva de $24 \mathrm{~h}$ observam-se valores significativamente inferiores aos normalmente usados.

Palavras-chave: chuva intensa, hidrologia, drenagem

\section{Relationships between intense rainfall of different durations for disaggregation of daily rainfall in Santa Catarina}

\section{ABSTRACT}

The aim of this study was to determine the relationships between rainfalls of different durations for the State of Santa Catarina. The series of annual maximum rainfall were defined with a duration of 5 to 1440 min of thirteen precipitation stations in Santa Catarina. For each duration maximum rainfall were estimated with a return period of 2 to 100 years, based on the Gumbel-Chow distribution. The relationship between the average rainfall of $24 \mathrm{~h}$ and one day rain does not depend on the return period, with an average of 1.17 for the State. When analyzing the time reading the daily precipitation it was messed that this ratio varies from 1.12 to 1.20 . In relation to $1 \mathrm{~h}$ of rain over $24 \mathrm{~h}$ of rain it was observed values significantly lower than those normally used.

Key words: intense rainfall, hydrology, drainage 


\section{INTRODUÇÃO}

O conhecimento das características da precipitação e sua relação no ciclo hidrológico são de suma importância para estudos estratégicos associados ao planejamento do meio ambiente (Carvalho \& Assad, 2005), à geração de energia e ao manejo da agricultura, especialmente em condições tropicais (Mello et al., 2003b).

No dimensionamento de obras de drenagem superficial é necessário conhecer as relações envolvendo a intensidadeduração-frequência (IDF) da chuva a ser utilizada no projeto; a utilização de equações de chuvas intensas e a forma mais usual para estimar a intensidade da chuva a ser aplicada na determinação de vazões de cheia. Para estimar os parâmetros de chuvas intensas utilizam-se séries históricas de dados de chuvas máximas com vários tempos de duração, extraídos de pluviogramas. As relações IDF são obtidas por meio de análises estatísticas de longas séries de dados observados em pluviógrafos (Silva et al., 1999; 2002; Back, 2006). No Brasil existe relativa facilidade de se obter dados de chuva de duração diária porém dados de chuvas de menor duração, devido à escassez de equipamentos registradores dificilmente são disponíveis e, quando existem, são séries relativamente curtas que apresentam muitas falhas nos registros de dados. Lima et al. (2005), Oliveira et al. (2008) e Back (2009) relatam a dificuldade de obtenção de longas séries de dados de precipitação, principalmente de registros pluviográficos. Cecílio \& Pruski (2003) destacam que a metodologia para determinar as relações IDF de chuvas exige exaustivo trabalho para tabulação, análise e interpretação de uma grande quantidade de pluviogramas.

Algumas metodologias foram desenvolvidas visando à obtenção das chuvas de menor duração, a partir dos dados pluviométricos diários. Bell (1969) apresentou relações para estimar a altura da chuva com duração de até 120 min a partir da chuva com duração de uma hora e período de retorno de 10 anos. Torrico (1975) desenvolveu a metodologia das isozonas, que pode ser aplicada em todo o território nacional. Damé et al. (2006) ajustaram as relações IDF com dados de precipitação estimadas por técnicas de desagregação de chuva diária. Damé et al. (2008) utilizaram séries sintéticas de precipitação para a estimativa das relações IDF mas dentre os métodos mais usados se destacam aqueles que se baseiam nas relações entre precipitações de diferentes durações para desagregar a chuva máxima diária em chuva com duração inferior. Trata-se de um método com a vantagem de ser de uso simples, de fornecer resultados satisfatórios e com grande similaridade para diferentes locais, o que lhe outorga validade regional. No Brasil são largamente empregadas as relações entre durações publicadas pela CETESB (1986), como os trabalhos de Mello et al. (2003a), Ferreira et al. (2005), Soprani \& Reis (2007) e Oliveira et al. (2008).

Segundo Genovez \& Zuffo (2000) as relações entre precipitações de diferentes durações apresentadas, além de utilizarem períodos muito curtos foram obtidas para uma média nacional. Esses valores médios nacionais deveriam ser revistos para os de médias regionais contemplando-se, desta forma, as precipitações predominantes em cada região. Os autores destacam, também, que esses valores necessitam ser atualizados, por se tratar de um estudo feito há muito tempo, baseado nas curvas de intensidade de precipitação-duraçãofrequência para 98 localidades do Brasil obtidas por Pfafstetter (1957) utilizando períodos de dados muito curtos sendo, para a maioria dos locais, da ordem de 10 anos de dados pluviográficos.

Este trabalho teve como objetivo determinar as relações entre precipitações de diferentes durações para o Estado de Santa Catarina como objetivo de desagregar a chuva diária em chuvas de curta duração.

\section{Material e MÉTODOS}

Foram utilizados os gráficos diários das estações meteorológicas do Instituto Nacional de Meteorologia (INMET) e da Empresa de Pesquisa Agropecuária e Extensão Rural de Santa Catarina (EPAGRI), com as localizações de acordo com as coordenadas da Tabela 1. Os pluviogramas foram digitalizados e se determinaram as séries de máximas anuais de chuva com duração de $5,10,15,20,25,30,45,50,55,60,75,90$ e 105 minutos e 2, 3, 4, 5, 6, 7, 8, 10, 12, 14, 16, 18, 20, 22 e $24 \mathrm{~h}$, além da chuva máxima de um dia, definida como o total da chuva acumulada diariamente, até as $9 \mathrm{~h}$.

Tabela 1. Estações pluviográficas utilizadas no trabalho

\begin{tabular}{lccccc}
\hline \multicolumn{1}{c}{ Estação } & $\begin{array}{c}\text { Latitude } \\
(\mathbf{S})\end{array}$ & $\begin{array}{c}\text { Longitude } \\
(\mathbf{W})\end{array}$ & $\begin{array}{c}\text { Altitude } \\
(\mathbf{m})\end{array}$ & $\begin{array}{c}\text { Período } \\
\text { de dados }\end{array}$ & $\begin{array}{c}\text { No de } \\
\text { anos }\end{array}$ \\
Chapecó & $27^{\circ} 07^{\prime}$ & $52^{\circ} 37^{\prime}$ & 679 & $1976-2005$ & 26 \\
Urussanga & $28^{\circ} 31^{\prime}$ & $49^{\circ} 19^{\prime}$ & 48 & $1986-2006$ & 24 \\
Campos Novos & $28^{\circ} 24^{\prime}$ & $51^{\circ} 12^{\prime}$ & 952 & $1984-2005$ & 21 \\
Florianópolis & $27^{\circ} 35^{\prime}$ & $48^{\circ} 34^{\prime}$ & 2 & $1984-2005$ & 22 \\
Lages & $27^{\circ} 49^{\prime}$ & $50^{\circ} 20^{\prime}$ & 937 & $1969-2005$ & 25 \\
Caçador & $26^{\circ} 46^{\prime}$ & $51^{\circ} 00^{\prime}$ & 960 & $1988-2005$ & 16 \\
Itajá & $26^{\circ} 54^{\prime}$ & $48^{\circ} 38^{\prime}$ & 5 & $1984-1999$ & 12 \\
Itá & $27^{\circ} 15^{\prime}$ & $52^{\circ} 21^{\prime}$ & 496 & $1981-2000$ & 16 \\
Ponte Serrada & $26^{\circ} 52^{\prime}$ & $52^{\circ} 01^{\prime}$ & 1100 & $1987-2000$ & 14 \\
Porto União & $26^{\circ} 14^{\prime}$ & $51^{\circ} 04^{\prime}$ & 778 & $1973-1992$ & 13 \\
Videira & $27^{\circ} 00^{\prime}$ & $51^{\circ} 09^{\prime}$ & 774 & $1986-2004$ & 19 \\
Laguna & $28^{\circ} 29^{\prime}$ & $48^{\circ} 48^{\prime}$ & 31 & $1970-1984$ & 12 \\
São J oaquim & $28^{\circ} 19^{\prime}$ & $49^{\circ} 55^{\prime}$ & 1288 & $1971-1987$ & 14 \\
\hline
\end{tabular}

A distribuição de Gumbel-Chow (Eltz et al., 1992) foi utilizada para a estimativa dos valores de chuva. Para cada duração foram estimados os valores de chuva com período de retorno de 2, 5, 10, 20, 25, 50 e 100 anos, por meio da Eq. (1).

$$
P_{T}=\bar{x}+\left(Y-Y_{n}\right) \frac{S}{S_{n}}
$$

em que:

$\mathrm{P}_{\mathrm{T}}$ - precipitação máxima com período de retorno $\mathrm{T}$ anos, $\mathrm{mm}$

$\overline{\mathrm{X}}$ - média da série de máximas anuais, $\mathrm{mm}$

$\mathrm{S}$ - desvio padrão da série de máximas anuais, $\mathrm{mm}$

Yn - média da variável reduzida

Sn - desvio padrão da variável reduzida

Y - variável reduzida calculada pela Eq. (2) 


$$
\mathrm{Y}=-\ln \left\{-\ln \left(1-\frac{1}{\mathrm{~T}}\right)\right\}
$$

em que:

$$
\mathrm{T} \text { - período de retorno, anos }
$$

A aderência das séries de máximas anuais à distribuição da Gumbel-Chow, foi testada pelo teste de Kolmogorov-Smirnov em nível de significância de 5\%, conforme Evangelista et al. (2006).

\section{RESULTADOS E DISCUSSÃO}

Na Tabela 2 constam os valores das relações entre precipitação máxima de 24 h e a precipitação máxima de um dia para o período de retorno de 2 a 100 anos. Comparando relações médias das diferentes estações, tem-se que a menor relação encontrada é de 1,11 para a estação de Chapecó a maior relação é de 1,24 para as estações de Florianópolis e Laguna, com média geral de 1,17 . Vários trabalhos mostram que a proporção entre a chuva máxima em 24 h e a chuva máxima de um dia é praticamente constante e independente do período de retorno. Occhipinti \& Santos (1966) utilizando séries de dados de 1928 a 1965 de São Paulo observaram a relação variando de 1,13 a 1,15 com média de 1,14 , valor este indicado pela CETESB e usado na maioria dos trabalhos, com desagregação de chuvas diárias, realizados no Brasil. Genovez \& Pegoraro (1998) obtiveram, analisando dados de 23 pluviógrafos do Estado de São Paulo, valor mínimo de 1,03 e máximo de 1,17, com média de 1,13. Torrico (1975) propõe, baseado nas estações usadas por Pfafstetter (1957) o valor 1,10 (1,095). com base em dados de vários pluviógrafos do Brasil, a CETESB (1986) recomendou o valor 1,14. Segundo Froehlich (1993), o U.S. Weather Bureau indicou o valor de 1,13 e Kessler \& Raad (1978) recomendaram o valor de 1,10. Weiss (1964), modelado em conceitos teóricos de probabilidade, estudou as relações entre precipitações médias obtidas por médias móveis e as precipitações obtidas em intervalos fixos, obtendo a relação de 1,143.
Na Tabela 3 constam as relações entre a precipitação máxima de $24 \mathrm{~h}$ e a precipitação máxima de um dia de duração, considerando-se os diferentes horários de leitura da precipitação diária. Observa-se que os valores variam de 1,03 a 1,49 e que, na maioria das estações, as maiores relações são obtidas quando a precipitação diária é computada entre as $7 \mathrm{e}$ $15 \mathrm{~h}$. Essas maiores relações observadas podem ser atribuídas, em parte, à ocorrência de chuvas convectivas nesses horários. Nerilo et al. (2002) afirmam que podem ocorrer precipitações máximas antes e depois do horário de observação, ocasionando o aumento do fator multiplicativo e que em regiões nas quais é passível haver chuvas convectivas devido ao aquecimento diurno, as chuvas mais intensas ocorrem ao final da tarde. $\mathrm{Na}$ Tabela 3 constam os valores das médias ponderadas pelo número de anos de observação de cada estação, verificandose que a média varia de 1,12 a 1,20. Nas estações meteorológicas a chuva de um dia é sempre medida em um horário fixo, normalmente às 9 (12 h de Greenwich) e nas estações da Agência Nacional de Águas (ANA) a leitura da precipitação é realizada, normalmente, às $07: 00 \mathrm{~h}$; já para os horários entre as 7 e $9 \mathrm{~h}$, a relação média é de 1,20.

Na Tabela 4 se apresentam as relações entre precipitações de diferentes durações observadas nas estações pluviográficas do Estado de Santa Catarina e na Tabela 5 constam as médias ponderadas dessas relações das estações de Santa Catarina e as relações estabelecidas para o Brasil (CETESB, 1986). Observa-se comportamento diferenciado nas estações de Florianópolis, Itajaí e Laguna, sobretudo nas relações entre a precipitação máxima de $1 \mathrm{~h}$ e a precipitação máxima de $24 \mathrm{~h}$, com valores de 0,$23 ; 0,24$ e 0,23 , respectivamente, muito inferiores aos das demais estações. Comparando-se as relações médias de Santa Catarina com as relações médias da CETESB (1986), observa-se que para as relações das chuvas com duração de 5 a $30 \mathrm{~min}$, as diferenças em relação aos valores indicados pela CETESB são inferiores a $4 \%$.

Segundo Monteiro \& Furtado (1995) há uma acentuada variação na precipitação pluviométrica dentro do Estado de Santa Catarina; em geral, a pluviosidade é menor na planície litorânea e os maiores valores de pluviosidade são observados próximos à encosta da Serra Geral. Na estreita área do litoral catarinense o

Tabela 2. Relação entre a precipitação máxima de 24 h e a precipitação máxima de 1 dia calculada com período de

\begin{tabular}{|c|c|c|c|c|c|c|c|c|}
\hline \multirow{2}{*}{ Estação } & \multicolumn{7}{|c|}{ T - Período de retorno (ano) } & \multirow{2}{*}{ Média } \\
\hline & 100 & 50 & 25 & 20 & 10 & 5 & 2 & \\
\hline Caçador & 1,14 & 1,14 & 1,15 & 1,15 & 1,15 & 1,16 & 1,18 & 1,15 \\
\hline Campos Novos & 1,23 & 1,22 & 1,22 & 1,21 & 1,20 & 1,19 & 1,16 & 1,20 \\
\hline Chapecó & 1,10 & 1,10 & 1,11 & 1,11 & 1,12 & 1,12 & 1,14 & 1,11 \\
\hline Florianópolis & 1,26 & 1,26 & 1,25 & 1,25 & 1,24 & 1,23 & 1,18 & 1,24 \\
\hline Itajaí & 1,14 & 1,14 & 1,15 & 1,15 & 1,15 & 1,15 & 1,17 & 1,15 \\
\hline Laguna & 1,27 & 1,26 & 1,26 & 1,25 & 1,24 & 1,23 & 1,18 & 1,24 \\
\hline Lages & 1,15 & 1,15 & 1,15 & 1,15 & 1,16 & 1,16 & 1,18 & 1,16 \\
\hline Itá & 1,10 & 1,10 & 1,11 & 1,11 & 1,11 & 1,12 & 1,14 & 1,11 \\
\hline Ponte Serrada & 1,17 & 1,17 & 1,17 & 1,16 & 1,16 & 1,15 & 1,13 & 1,16 \\
\hline Porto União & 1,21 & 1,21 & 1,20 & 1,20 & 1,19 & 1,18 & 1,17 & 1,19 \\
\hline Urussanga & 1,13 & 1,13 & 1,13 & 1,13 & 1,13 & 1,14 & 1,15 & 1,13 \\
\hline Videira & 1,16 & 1,16 & 1,15 & 1,15 & 1,14 & 1,14 & 1,12 & 1,15 \\
\hline São J oaquim & 1,17 & 1,17 & 1,17 & 1,17 & 1,16 & 1,16 & 1,15 & 1,17 \\
\hline Média & 1,17 & 1,17 & 1,17 & 1,17 & 1,17 & 1,16 & 1,16 & 1,17 \\
\hline
\end{tabular}
retorno de 2 a 100 anos 
Tabela 3. Relação entre a preci pitação máxima de 24 h e a precipitação máxima de 1 dia para as diferentes horas de leitura da precipitação diária

\begin{tabular}{|c|c|c|c|c|c|c|c|c|c|c|c|c|c|c|}
\hline Hora & Caç. & $\mathrm{CN}$ & Cha. & Flo. & Itajaí & Lag. & Lages & Itá & PS & PU & Uru. & Vid. & SJ & Média \\
\hline 01:00 & 1,15 & 1,22 & 1,07 & 1,34 & 1,08 & 1,30 & 1,14 & 1,05 & 1,24 & 1,03 & 1,04 & 1,18 & 1,18 & 1,14 \\
\hline 02:00 & 1,16 & 1,19 & 1,08 & 1,40 & 1,09 & 1,43 & 1,16 & 1,08 & 1,24 & 1,04 & 1,05 & 1,17 & 1,20 & 1,16 \\
\hline 03:00 & 1,14 & 1,19 & 1,10 & 1,39 & 1,13 & 1,49 & 1,14 & 1,10 & 1,22 & 1,05 & 1,05 & 1,17 & 1,22 & 1,15 \\
\hline 04:00 & 1,14 & 1,19 & 1,13 & 1,36 & 1,17 & 1,42 & 1,13 & 1,08 & 1,18 & 1,08 & 1,05 & 1,17 & 1,24 & 1,15 \\
\hline 05:00 & 1,15 & 1,21 & 1,10 & 1,37 & 1,20 & 1,40 & 1,13 & 1,10 & 1,15 & 1,14 & 1,07 & 1,17 & 1,24 & 1,16 \\
\hline 06:00 & 1,16 & 1,21 & 1,10 & 1,38 & 1,21 & 1,39 & 1,16 & 1,11 & 1,15 & 1,21 & 1,09 & 1,17 & 1,24 & 1,17 \\
\hline 07:00 & 1,20 & 1,24 & 1,11 & 1,32 & 1,22 & 1,34 & 1,16 & 1,10 & 1,12 & 1,26 & 1,09 & 1,16 & 1,20 & 1,18 \\
\hline 08:00 & 1,22 & 1,25 & 1,15 & 1,30 & 1,24 & 1,31 & 1,15 & 1,10 & 1,15 & 1,30 & 1,12 & 1,16 & 1,19 & 1,19 \\
\hline 09:00 & 1,26 & 1,25 & 1,17 & 1,28 & 1,30 & 1,29 & 1,15 & 1,14 & 1,17 & 1,30 & 1,13 & 1,15 & 1,17 & 1,20 \\
\hline $10: 00$ & 1,27 & 1,22 & 1,17 & 1,27 & 1,31 & 1,28 & 1,16 & 1,19 & 1,20 & 1,26 & 1,14 & 1,16 & 1,17 & 1,20 \\
\hline 11:00 & 1,22 & 1,20 & 1,19 & 1,23 & 1,29 & 1,27 & 1,17 & 1,18 & 1,20 & 1,26 & 1,17 & 1,11 & 1,17 & 1,20 \\
\hline $12: 00$ & 1,14 & 1,17 & 1,21 & 1,20 & 1,20 & 1,26 & 1,14 & 1,18 & 1,19 & 1,34 & 1,20 & 1,12 & 1,14 & 1,19 \\
\hline 13:00 & 1,12 & 1,15 & 1,18 & 1,17 & 1,17 & 1,25 & 1,13 & 1,17 & 1,16 & 1,44 & 1,24 & 1,12 & 1,11 & 1,18 \\
\hline $14: 00$ & 1,09 & 1,11 & 1,15 & 1,16 & 1,14 & 1,17 & 1,12 & 1,18 & 1,15 & 1,38 & 1,25 & 1,13 & 1,12 & 1,16 \\
\hline 15:00 & 1,05 & 1,11 & 1,12 & 1,12 & 1,13 & 1,04 & 1,13 & 1,18 & 1,10 & 1,32 & 1,29 & 1,12 & 1,10 & 1,15 \\
\hline $16: 00$ & 1,06 & 1,12 & 1,10 & 1,07 & 1,11 & 1,03 & 1,14 & 1,15 & 1,08 & 1,30 & 1,28 & 1,10 & 1,11 & 1,14 \\
\hline $17: 00$ & 1,07 & 1,18 & 1,10 & 1,10 & 1,08 & 1,03 & 1,16 & 1,12 & 1,07 & 1,35 & 1,24 & 1,10 & 1,11 & 1,14 \\
\hline 18:00 & 1,13 & 1,27 & 1,10 & 1,13 & 1,07 & 1,04 & 1,17 & 1,10 & 1,07 & 1,21 & 1,20 & 1,10 & 1,12 & 1,15 \\
\hline 19:00 & 1,16 & 1,27 & 1,07 & 1,16 & 1,07 & 1,11 & 1,19 & 1,10 & 1,10 & 1,13 & 1,18 & 1,12 & 1,11 & 1,15 \\
\hline 20:00 & 1,18 & 1,25 & 1,05 & 1,18 & 1,08 & 1,16 & 1,22 & 1,06 & 1,12 & 1,10 & 1,13 & 1,14 & 1,10 & 1,14 \\
\hline $21 ; 00$ & 1,17 & 1,25 & 1,08 & 1,21 & 1,06 & 1,16 & 1,20 & 1,06 & 1,15 & 1,07 & 1,09 & 1,18 & 1,17 & 1,14 \\
\hline $22: 00$ & 1,13 & 1,22 & 1,07 & 1,19 & 1,09 & 1,17 & 1,19 & 1,05 & 1,18 & 1,05 & 1,05 & 1,18 & 1,19 & 1,13 \\
\hline 23:00 & 1,13 & 1,22 & 1,07 & 1,19 & 1,09 & 1,20 & 1,16 & 1,06 & 1,20 & 1,03 & 1,03 & 1,17 & 1,18 & 1,12 \\
\hline $24: 00$ & 1,16 & 1,22 & 1,07 & 1,22 & 1,09 & 1,23 & 1,16 & 1,05 & 1,22 & 1,03 & 1,03 & 1,18 & 1,17 & 1,13 \\
\hline Média & 1,15 & 1,20 & 1,11 & 1,24 & 1,15 & 1,24 & 1,16 & 1,11 & 1,16 & 1,19 & 1,13 & 1,15 & 1,17 & 1,16 \\
\hline
\end{tabular}

Caç. - Caçador; CN - Campos Novos; Cha. - Chapecó; Flo. - Florianópolis; Lag. - Laguna; PS - Ponte Serrada; PU - Porto União; Uru. - Urussanga; Vid. - Videira; SJ - São J oaquim

Tabela 4. Relações entre precipitações de diferentes durações observadas nas estações pluviográficas do Estado de Santa Catarina

\begin{tabular}{|c|c|c|c|c|c|c|c|c|c|c|c|c|c|}
\hline \multirow{2}{*}{$\begin{array}{l}\text { Relações entre } \\
\text { durações }\end{array}$} & \multicolumn{13}{|c|}{ Estação pluviográfica } \\
\hline & Caç. & $\mathrm{CN}$ & Cha. & Flo. & Itajaí & Lag. & Lages & Itá & PS & PU & Uru. & Vid. & SJ \\
\hline $5 \mathrm{~min} / 30 \mathrm{~min}$ & 0,37 & 0,36 & 0,36 & 0,36 & 0,51 & 0,34 & 0,29 & 0,32 & 0,38 & 0,36 & 0,31 & 0,32 & 0,44 \\
\hline $10 \mathrm{~min} / 30 \mathrm{~min}$ & 0,52 & 0,56 & 0,53 & 0,53 & 0,57 & 0,52 & 0,48 & 0,49 & 0,56 & 0,59 & 0,48 & 0,51 & 0,57 \\
\hline $15 \mathrm{~min} / 30 \mathrm{~min}$ & 0,69 & 0,72 & 0,67 & 0,65 & 0,66 & 0,69 & 0,63 & 0,64 & 0,70 & 0,78 & 0,68 & 0,66 & 0,69 \\
\hline $20 \mathrm{~min} / 30 \mathrm{~min}$ & 0,81 & 0,82 & 0,79 & 0,78 & 0,79 & 0,84 & 0,76 & 0,81 & 0,83 & 0,86 & 0,81 & 0,82 & 0,82 \\
\hline $25 \mathrm{~min} / 30 \mathrm{~min}$ & 0,93 & 0,91 & 0,90 & 0,89 & 0,87 & 0,93 & 0,88 & 0,92 & 0,93 & 0,93 & 0,92 & 0,90 & 0,91 \\
\hline $30 \mathrm{~min} / 1 \mathrm{~h}$ & 0,71 & 0,73 & 0,79 & 0,63 & 0,67 & 0,66 & 0,76 & 0,80 & 0,71 & 0,84 & 0,79 & 0,77 & 0,83 \\
\hline $35 \mathrm{~min} / 1 \mathrm{~h}$ & 0,75 & 0,79 & 0,84 & 0,70 & 0,71 & 0,71 & 0,83 & 0,86 & 0,76 & 0,90 & 0,85 & 0,83 & 0,89 \\
\hline $40 \mathrm{~min} / 1 \mathrm{~h}$ & 0,80 & 0,83 & 0,87 & 0,76 & 0,75 & 0,76 & 0,88 & 0,89 & 0,82 & 0,96 & 0,89 & 0,87 & 0,91 \\
\hline $45 \mathrm{~min} / 1 \mathrm{~h}$ & 0,86 & 0,87 & 0,92 & 0,83 & 0,79 & 0,81 & 0,91 & 0,92 & 0,87 & 0,97 & 0,94 & 0,91 & 0,93 \\
\hline $50 \mathrm{~min} / 1 \mathrm{~h}$ & 0,91 & 0,91 & 0,95 & 0,90 & 0,82 & 0,87 & 0,93 & 0,94 & 0,90 & 0,98 & 0,96 & 0,95 & 0,96 \\
\hline $55 \mathrm{~min} / 1 \mathrm{~h}$ & 0,97 & 0,95 & 0,98 & 0,95 & 0,93 & 0,94 & 0,97 & 0,97 & 0,95 & 0,98 & 0,98 & 0,98 & 0,98 \\
\hline $1 \mathrm{~h} / 24 \mathrm{~h}$ & 0,33 & 0,44 & 0,37 & 0,23 & 0,24 & 0,23 & 0,39 & 0,45 & 0,41 & 0,32 & 0,35 & 0,39 & 0,31 \\
\hline $1,25 \mathrm{~h} / 24 \mathrm{~h}$ & 0,36 & 0,48 & 0,40 & 0,26 & 0,27 & 0,26 & 0,43 & 0,49 & 0,44 & 0,35 & 0,36 & 0,42 & 0,33 \\
\hline $1,50 h / 24 h$ & 0,39 & 0,50 & 0,45 & 0,29 & 0,32 & 0,28 & 0,47 & 0,53 & 0,46 & 0,39 & 0,38 & 0,46 & 0,34 \\
\hline $1,75 \mathrm{~h} / 24 \mathrm{~h}$ & 0,42 & 0,53 & 0,50 & 0,33 & 0,35 & 0,30 & 0,49 & 0,55 & 0,49 & 0,43 & 0,40 & 0,49 & 0,35 \\
\hline $2 \mathrm{~h} / 24 \mathrm{~h}$ & 0,46 & 0,55 & 0,53 & 0,35 & 0,38 & 0,31 & 0,52 & 0,57 & 0,51 & 0,45 & 0,41 & 0,51 & 0,35 \\
\hline $2,5 \mathrm{~h} / 24 \mathrm{~h}$ & 0,50 & 0,56 & 0,57 & 0,38 & 0,40 & 0,32 & 0,54 & 0,60 & 0,53 & 0,48 & 0,44 & 0,53 & 0,35 \\
\hline $3 \mathrm{~h} / 24 \mathrm{~h}$ & 0,54 & 0,58 & 0,60 & 0,40 & 0,44 & 0,35 & 0,55 & 0,63 & 0,57 & 0,53 & 0,46 & 0,55 & 0,36 \\
\hline $4 h / 24 h$ & 0,58 & 0,61 & 0,62 & 0,44 & 0,48 & 0,40 & 0,60 & 0,68 & 0,62 & 0,56 & 0,52 & 0,58 & 0,38 \\
\hline $5 \mathrm{~h} / 24 \mathrm{~h}$ & 0,59 & 0,63 & 0,67 & 0,47 & 0,50 & 0,43 & 0,62 & 0,71 & 0,63 & 0,58 & 0,55 & 0,61 & 0,41 \\
\hline $6 \mathrm{~h} / 24 \mathrm{~h}$ & 0,61 & 0,64 & 0,70 & 0,53 & 0,54 & 0,48 & 0,64 & 0,74 & 0,66 & 0,59 & 0,61 & 0,64 & 0,43 \\
\hline $7 \mathrm{~h} / 24 \mathrm{~h}$ & 0,63 & 0,65 & 0,73 & 0,56 & 0,57 & 0,51 & 0,65 & 0,76 & 0,68 & 0,62 & 0,66 & 0,66 & 0,46 \\
\hline $8 \mathrm{~h} / 24 \mathrm{~h}$ & 0,64 & 0,66 & 0,75 & 0,60 & 0,59 & 0,53 & 0,67 & 0,77 & 0,70 & 0,65 & 0,70 & 0,69 & 0,51 \\
\hline $10 \mathrm{~h} / 24 \mathrm{~h}$ & 0,70 & 0,68 & 0,81 & 0,64 & 0,66 & 0,60 & 0,70 & 0,80 & 0,73 & 0,71 & 0,76 & 0,70 & 0,61 \\
\hline $12 \mathrm{~h} / 24 \mathrm{~h}$ & 0,76 & 0,71 & 0,86 & 0,72 & 0,71 & 0,65 & 0,75 & 0,84 & 0,76 & 0,74 & 0,83 & 0,77 & 0,68 \\
\hline $14 \mathrm{~h} / 24 \mathrm{~h}$ & 0,80 & 0,74 & 0,89 & 0,78 & 0,78 & 0,73 & 0,79 & 0,88 & 0,78 & 0,77 & 0,87 & 0,81 & 0,74 \\
\hline $16 \mathrm{~h} / 24 \mathrm{~h}$ & 0,84 & 0,78 & 0,91 & 0,86 & 0,82 & 0,81 & 0,84 & 0,90 & 0,81 & 0,86 & 0,91 & 0,87 & 0,79 \\
\hline $18 \mathrm{~h} / 24 \mathrm{~h}$ & 0,87 & 0,81 & 0,93 & 0,91 & 0,86 & 0,85 & 0,89 & 0,91 & 0,85 & 0,91 & 0,93 & 0,91 & 0,85 \\
\hline $20 \mathrm{~h} / 24 \mathrm{~h}$ & 0,92 & 0,85 & 0,96 & 0,94 & 0,91 & 0,88 & 0,92 & 0,95 & 0,90 & 0,95 & 0,96 & 0,95 & 0,89 \\
\hline $22 \mathrm{~h} / 24 \mathrm{~h}$ & 0,97 & 0,96 & 0,97 & 0,96 & 0,95 & 0,97 & 0,97 & 0,98 & 0,96 & 0,98 & 0,98 & 0,99 & 0,95 \\
\hline $24 \mathrm{~h} / 1 \mathrm{dia}$ & 1,15 & 1,20 & 1,11 & 1,24 & 1,15 & 1,23 & 1,16 & 1,11 & 1,16 & 1,19 & 1,13 & 1,15 & 1,17 \\
\hline
\end{tabular}


Tabela 5. Relações médias entre precipitações de diferentes durações observadas nas estações pluviográficas de Santa Catarina

\begin{tabular}{|c|c|c|c|c|}
\hline Relação & SC & SC interior ${ }^{1}$ & SC litoral $^{2}$ & CETESB \\
\hline $5 \mathrm{~min} / 30 \mathrm{~min}$ & 0,35 & 0,34 & 0,39 & 0,34 \\
\hline $10 \mathrm{~min} / 30 \mathrm{~min}$ & 0,53 & 0,52 & 0,54 & 0,54 \\
\hline $15 \mathrm{~min} / 30 \mathrm{~min}$ & 0,68 & 0,68 & 0,66 & 0,70 \\
\hline $20 \mathrm{~min} / 30 \mathrm{~min}$ & 0,81 & 0,81 & 0,80 & 0,81 \\
\hline $25 \mathrm{~min} / 30 \mathrm{~min}$ & 0,91 & 0,91 & 0,90 & 0,91 \\
\hline $30 \mathrm{~min} / 1 \mathrm{~h}$ & 0,75 & 0,77 & 0,65 & 0,74 \\
\hline $35 \mathrm{~min} / 1 \mathrm{~h}$ & 0,80 & 0,83 & 0,70 & - \\
\hline $40 \mathrm{~min} / 1 \mathrm{~h}$ & 0,85 & 0,87 & 0,76 & - \\
\hline $45 \mathrm{~min} / 1 \mathrm{~h}$ & 0,89 & 0,91 & 0,82 & - \\
\hline $50 \mathrm{~min} / 1 \mathrm{~h}$ & 0,93 & 0,94 & 0,87 & - \\
\hline $55 \mathrm{~min} / 1 \mathrm{~h}$ & 0,96 & 0,97 & 0,94 & - \\
\hline $1 \mathrm{~h} / 24 \mathrm{~h}$ & 0,35 & 0,38 & 0,23 & 0,42 \\
\hline $1,25 \mathrm{~h} / 24 \mathrm{~h}$ & 0,38 & 0,41 & 0,27 & - \\
\hline $1,50 h / 24 h$ & 0,41 & 0,44 & 0,30 & - \\
\hline $1,75 \mathrm{~h} / 24 \mathrm{~h}$ & 0,44 & 0,47 & 0,32 & - \\
\hline $2 \mathrm{~h} / 24 \mathrm{~h}$ & 0,46 & 0,49 & 0,34 & - \\
\hline $2,5 \mathrm{~h} / 24 \mathrm{~h}$ & 0,49 & 0,52 & 0,37 & - \\
\hline $3 \mathrm{~h} / 24 \mathrm{~h}$ & 0,51 & 0,54 & 0,40 & - \\
\hline $4 h / 24 h$ & 0,55 & 0,58 & 0,44 & - \\
\hline $5 \mathrm{~h} / 24 \mathrm{~h}$ & 0,58 & 0,61 & 0,47 & - \\
\hline $6 \mathrm{~h} / 24 \mathrm{~h}$ & 0,61 & 0,63 & 0,52 & 0,72 \\
\hline $7 \mathrm{~h} / 24 \mathrm{~h}$ & 0,64 & 0,66 & 0,55 & - \\
\hline $8 \mathrm{~h} / 24 \mathrm{~h}$ & 0,66 & 0,68 & 0,58 & - \\
\hline $10 \mathrm{~h} / 24 \mathrm{~h}$ & 0,71 & 0,73 & 0,63 & 0,82 \\
\hline $12 \mathrm{~h} / 24 \mathrm{~h}$ & 0,76 & 0,78 & 0,70 & 0,85 \\
\hline $14 \mathrm{~h} / 24 \mathrm{~h}$ & 0,81 & 0,81 & 0,77 & - \\
\hline $16 \mathrm{~h} / 24 \mathrm{~h}$ & 0,85 & 0,85 & 0,84 & - \\
\hline $18 \mathrm{~h} / 24 \mathrm{~h}$ & 0,89 & 0,89 & 0,88 & - \\
\hline $20 \mathrm{~h} / 24 \mathrm{~h}$ & 0,93 & 0,93 & 0,92 & - \\
\hline $22 \mathrm{~h} / 24 \mathrm{~h}$ & 0,97 & 0,97 & 0,96 & - \\
\hline 24 h/1 dia & 1,16 & 1,18 & 1,24 & 1,14 \\
\hline
\end{tabular}

${ }_{1}^{1}$ Média das relações observadas em todas as estações, exceto Florianópolis, Itajaí e Laguna 2 Médias das relações observadas nas estações de Florianópolis, Itajaí e Laguna

máximo pluviométrico ocorre no verão enquanto o índice mínimo é, na maioria das vezes, registrado no inverno e, secundariamente, no outono. Essas diferenças podem ser devidas à atuação diferenciada das massas de ar na região sul do País, refletindo diretamente na frequência e na intensidade das chuvas. Assis (1993) destacou que a atuação diferenciada das massas de ar é responsável pelas diferenças no padrão pluviométrico verificadas na região sul e sudeste do Brasil. Monteiro (2007) afirma que os diversos sistemas que atuam no Sul do Brasil imprimem, a Santa Catarina, uma dinâmica climática bastante acentuada, com boa distribuição de chuvas no decorrer do ano, tendo em vista que todos os sistemas instáveis são produtores de chuva. As massas de ar tropical continental ocorrem na região Sul e de forma restrita, no verão. A frente fria é o sistema atmosférico que mais interfere nas condições de tempo na Região Sul, o ano inteiro. Embora as frentes frias ocorram durante o ano todo, observa-se que a atuação desses sistemas se dá de forma diferente nas estações do ano e também entre o litoral e o interior do Estado. No verão, a atuação das frentes frias é maior sobre o Oceano Atlântico; no outono adentram mais pelo continente e no inverno, com o continente mais frio, as massas de ar provenientes de grandes latitudes se tornam mais importantes na distribuição de precipitação na Região Sul e, embora o número médio de incursões mensais seja igual, no inverno as frentes frias possuem atuação mais continental.
Monteiro \& Furtado (1995) comentam que o relevo de Santa Catarina contribui fundamentalmente na distribuição diferenciada da precipitação em distintas áreas do Estado, sendo as precipitações mais abundantes nas áreas mais próximas às encostas de montanha, pois a elevação do ar úmido e quente favorece o aumento do volume de precipitações. Neste sentido são observados índices de precipitação maiores nos municípios próximos à encosta da Serra Geral, quando comparados com a zona costeira. Do Planalto ao Oeste do Estado as chuvas são mais volumosas que em áreas próximas ao litoral. Tal fato é explicado por Monteiro (2001) pela influência da Baixa do Chaco não organização de condições de tempo mais instáveis, associada às frentes frias. Essas condições de tempo formadas por pancadas de chuva e trovoada, atingem com mais intensidade as regiões do Oeste, Meio-Oeste e Planalto. O autor ressalta, também, que no outono as precipitações registradas estão associadas mais à passagem de frentes frias que a chuvas convectivas. $\mathrm{Na}$ passagem do outono para o inverno, entre os meses de junho e agosto, a precipitação aumenta na Serra Geral e diminui intensamente na faixa litorânea, comportamento que deve-se ao sentido de deslocamento das frentes frias, normalmente de sudoeste-nordeste. Desta forma, as frentes frias atuam com maior intensidade sobre o planalto gaúcho e catarinense. Ao cruzarem as escarpas da Serra Geral, as frentes frias se tornam estáveis e causam menor pluviosidade nas áreas próximas ao litoral. Segundo Monteiro (2007), este aumento dos índices pluviométricos na primavera está associado à atuação sobre a região dos Complexos Convectivos de Mesoescala (CCM), fenômenos que se formam, em sua maioria, sobre o norte da Argentina e se deslocam para o leste, atingindo o Estado de Santa Catarina, favorecendo a ocorrência de pancadas de chuva forte com trovoada e granizo isolado. A influência dos CCM é mais visível no interior do Estado. Minuzzi (2010) mostra a influência do El Ninõ Oscilação Sul no comportamento da chuva em Santa Catarina nas diferentes estações do ano.

Também Lobo \& Magni (1987) apresentam os valores médios obtidos para 11 cidades do Estado de São Paulo muito próximos aos obtidos para Santa Catarina. Já para a duração de $1 \mathrm{~h}$ a média estimada com as estações de Santa Catarina $(0,360)$ é $15 \%$ inferior ao valor estabelecido pela $\operatorname{CETESB}(0,42)$. Lobo \& Magni (1987) citam a relação média de 0,51. Das treze estações estudadas somente foi observada a relação 0,42 nas estações de Canoinhas e Itá, localizadas no planalto catarinense; para as durações superiores a $1 \mathrm{~h}$, os valores médios observados em Santa Catarina são inferiores aos das relações citadas pela CETESB(1986).

No processo de desagregação das chuvas diárias o primeiro passo é estabelecer a chuva de $24 \mathrm{~h}$ e depois as chuvas com duração inferior a cada etapa,multiplicando-se os coeficientes. Desta forma, os erros na estimativa das chuvas se vão acumulando a cada coeficiente utilizado, de tal modo que o uso do coeficiente médio de Santa Catarina determina diferenças acima de até $40 \%$ nas estimativas de chuvas com duração inferior a 30 min para as estações de Florianópolis, Itajaí e Laguna. Para diminuir esses erros foram calculadas as relações médias para as estações de Florianópolis, Itajaí e Laguna, denominadas SC litoral, e a média para as demais estações, denominadas SC 


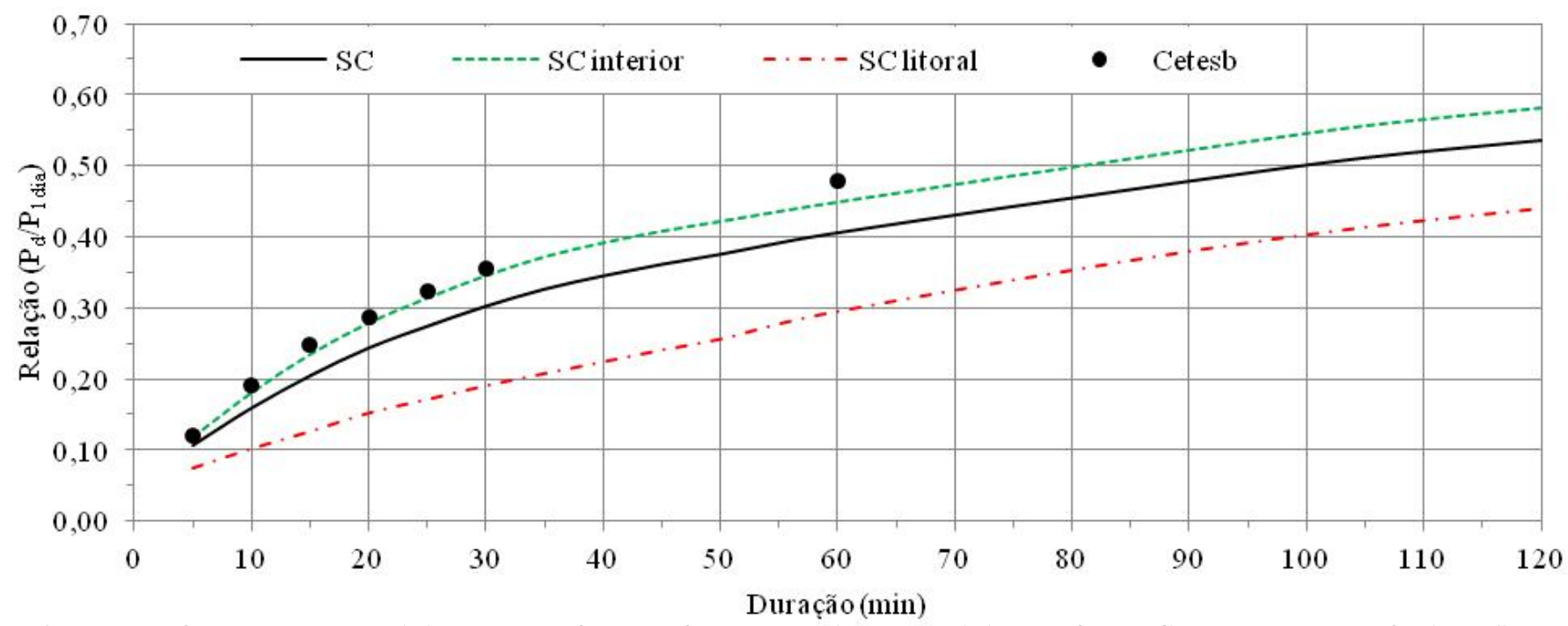

Figura 1. Relação entre a preci pitação com duração de 5 a 120 min e a precipitação de um dia, para estações pluviográficas de Santa Catarina

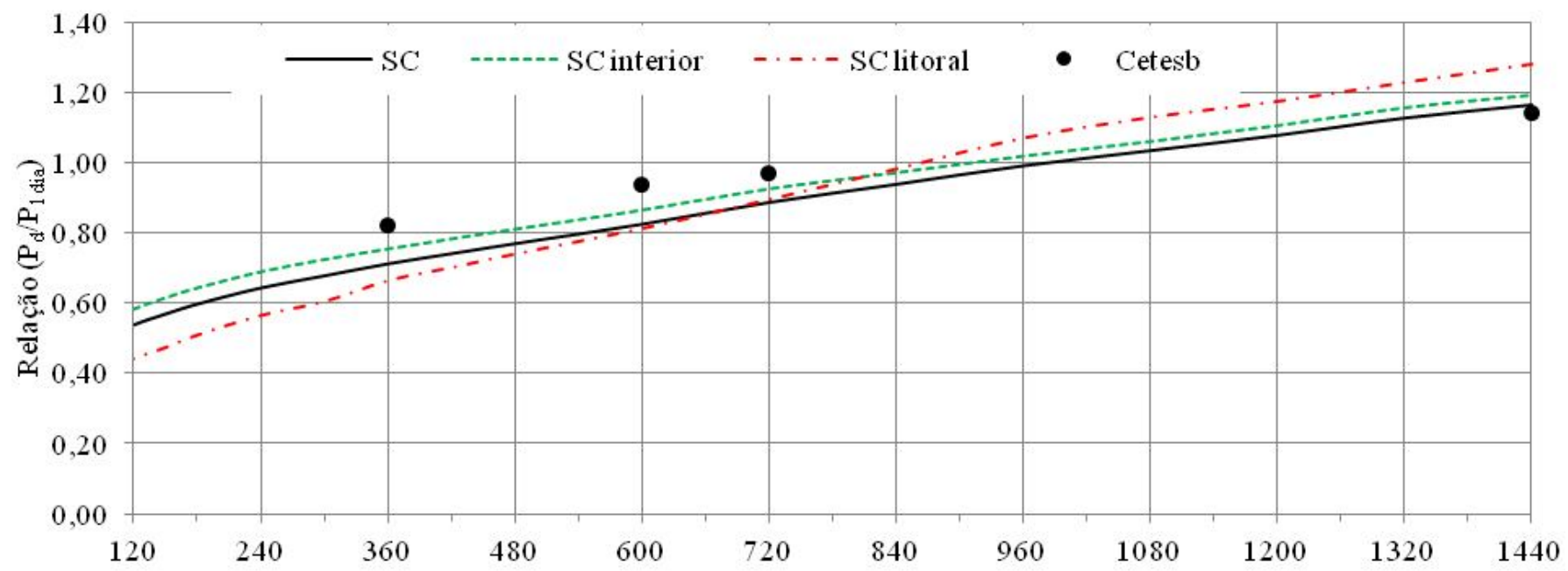

Duração (min)

Figura 2. Relação entre a precipitação com duração de 120 a 1440 min e a precipitação de um dia para estações pluviográficas de Santa Catarina

interior. Nas Figuras 1 e 2 estão representadas as relações entre a precipitação com duração "d" minutos $\left(\mathrm{P}_{\mathrm{d}}\right)$ e a precipitação máxima de um dia $\left(\mathrm{P}_{1 \mathrm{dia}}\right)$. Observa-se que as estações $\mathrm{SC}$ interior apresentam relações muito próximas às estabelecidas pela CETESB (1986), com diferenças inferiores a 6\%. O uso do valor 1,18 para a relação entre a precipitação máxima de $24 \mathrm{~h}$ e a precipitação de um dia, compensa os menores valores dos coeficientes obtidos para as durações entre 1 a 24 h; já as estações SC litoral apresentam relações inferiores às relações da CETESB para durações de até $720 \mathrm{~min}$, e superiores para as durações entre 720 e 1440 min.

Comparando-se as relações SC litoral com as relações da CETESB, observa-se que as diferenças são significativas podendo a diferença atingir $50 \%$ na duração de $15 \mathrm{~min}$. A utilização das relações médias para SC interior e SC litoral, permite reduzir os erros a valores abaixo de $10 \%$ quando comparadas aos valores de cada estação. Genovez \& Zuffo (2000) já mostraram que os coeficientes médios obtidos para o
Brasil podem gerar erros nas estimativas acima de $30 \%$ quando comparados com as estimativas baseadas em dados de pluviógrafos dos locais e, por isto, tal metodologia deve ser aplicada em localidades em que não há disponibilidade de registros pluviográficos.

\section{CONCLusÕES}

1. As relações entre precipitações máximas de $24 \mathrm{~h}$ e as máximas de um dia calculadas com período de retorno de 2 a 100 anos nas estações pluviográficas de Santa Catarina, praticamente se mantêm constantes e com média de 1,17.

2. As relações entre precipitações máximas de $24 \mathrm{~h}$ e as máximas de um dia, variam de acordo com a hora da leitura da precipitação diária sendo que, em média, variam de 1,12 a 1,20; para a chuva média diária medida entre as 7 e $9 \mathrm{~h}$ da manhã, a relação média para Santa Catarina é de 1,20. 
3. As relações entre precipitações de diferentes durações ajustadas para as estações do interior do Estado de Santa Catarina apresentam diferenças inferiores a 5\% em relação aos valores estabelecidos pela CETESB, enquanto as relações médias das estações do litoral apresentam valores de até 50\% inferiores aos das relações estabelecidas pela CETESB.

\section{LITERATURA CITADA}

Assis, F. N. de. Ajuste da função gama aos totais semanais de chuva de Pelotas-RS. Revista Brasileira de Agrometeorologia, v.1, p.131-136, 1993.

Back, A. J. Relações intensidade-duração-freqüência de chuvas intensas de Chapecó, Estado de Santa Catarina. Acta Scientiarum, Agronomy, v.28, p.575-581, 2006.

Back, A. J. Relações entre precipitação intensas de diferentes durações ocorridas no município de Urussanga, SC. Revista Brasileira de Engenharia Agrícola e Ambiental, v.13, p.170175, 2009.

Bell, F. G. Generalized rainfall-duration-frequency relationships. Journal of Hydraulics Division, v.95, p311-327, 1969.

Carvalho, J. P.; Assad, E. D. Análise espacial da precipitação pluviométrica no Estado de São Paulo: Comparação de métodos de interpolação. Revista Engenharia Agrícola, v.25, p.377-384, 2005.

Cecílio, R. A.; Pruski, F.F. Interpolação dos parâmetros de equações de chuvas intensas com uso do inverso de potências da distância. Revista Brasileira de Engenharia Agrícola e Ambiental, v.7, p.501-504, 2003.

CETESB - Companhia de Tecnologia de Saneamento Ambiental. Drenagem urbana - Manual de projeto. 3.ed. São Paulo: CETESB, 1986. 464p.

Damé, R. C. F.; Pedrotti, C. B. M.; Cardoso, M. A.; Silveira, C. P.; Duarte, L. A.; Moreira, A. C. Comparação entre curvas intensidade-duração-frequência de ocorrência de precipitação obtidas a partir de dados pluviográficos com aquelas estimadas por técnicas de desagregação de chuva diária. Revista Brasileira de Agrociência, v.12, p.505-509, 2006.

Damé, R. C. F.; Teixeira, C. F. A.; Terra, V. S. S. Comparação de diferentes metodologias para estimativa de curvas intensidade-duração-frequência para Pelotas-RS. Revista Engenharia Agrícola, v.28, p.245-255, 2008.

Eltz, F. L.; Reichert, J. M.; Cassol, E. A. Período de retorno de chuvas em Santa Maria, RS. Revista Brasileira de Ciência do Solo, v.16, p.265-269, 1992.

Evangelista, A. W. P.; Carvalho, L. D.; Dantas, A. A. A. Bernardino, D. T. Potencial erosivo das chuvas em LavrasMG: Distribuição, probabilidade de ocorrência e período de retorno. Irriga, v.11, p.1-11, 2006.

Ferreira, J. C.; Daniel, L. A.; Tomazela, M. Parâmetros para equações mensais de estimativas de precipitação de intensidade máxima para o Estado de São Paulo - Fase I. Revista Ciência e Agrotecnologia, v.29, p.1175-1187, 2005.
Froehlich, D. C. Short-duration-rainfall intensity equations for drainage design. Journal of Irrigation and Drainage Engineering, v.119, p.814-828, 1993.

Genovez, A. M.; Pegoraro, R. C. F. Análise e avaliação de equações de chuvas intensas generalizadas. In: Congresso Latinoamericano de Hidráulica 18, 1998, Oaxaca. Anais... Oaxaca: IAHR, v.1, 1998, p.369-379.

Genovez, A. M.; Zuffo, A. C. Chuvas intensas no Estado de São Paulo: Estudos existentes e análise comparativa. Revista Brasileira de Recursos Hídricos, v.5, p.45-58, 2000.

Kessler, J.; Raad, S. J. Análise de dados pluviométricos. In: International Institute for Land Reclamation and Improvement. Principios y aplicaciones del drenaje. Wageningen: ILRI. v.3, 1978, p.16-57, Publication 16.

Lima, H. M. F.; Mata, I. P.; Lima, A. V. F. Aplicação e validação de um simulador estocástico de variáveis climáticas: O caso da precipitação. Revista Ingenieria del Agua, v.12, p.27-37, 2005.

Lobo, G. A.; Magni, N. L. G. Estudo sobre proporções entre chuvas intensas de diferentes durações. In: VII Simpósio Brasileiro de Hidrologia e Recursos Hídricos. Anais... Salvador: ABRH, v.2, 1987, p.103-114.

Mello, C. R.; Lima, J. M.; Silva, A. M.; Mello, J. M.; Oliveira, M. S. Krigagem e inverso do quadrado da distância para interpolação dos parâmetros da equação de chuvas intensas. Revista Brasileira de Ciência do Solo, v.27, p.925-933, 2003a.

Mello, C. R.; Silva, A. M.; Lima, J. M.; Ferreira, D. F.; Oliveira, M. S. Modelos matemáticos para predição da chuva de projeto para regiões do Estado de Minas Gerais. Revista Brasileira de Engenharia Agrícola e Ambiental, v.7, p.121$128,2003 \mathrm{~b}$.

Minuzzi, R. B.; Chuvas em Santa Catarina durante eventos do El Niño oscilação sul. Geosul, v.25, p.107-127, 2010.

Monteiro, M. A. Caracterização climática do Estado de Santa Catarina: uma abordagem dos principais sistemas atmosféricos que atua durante o ano. Geosul, v.16, p.69-78, 2001.

Monteiro, M. A. Dinâmica atmosférica e a caracterização dos tipos de tempo na Bacia Hidrográfica do Rio Araranguá, Florianópolis: UFSC, 2007. 224p. Tese Doutorado

Monteiro, M. A.; Furtado, S. M. O clima do trecho Florianópolis - Porto Alegre: Uma abordagem dinâmica. Geosul, v.10, p.117-133, 1995.

Nerilo, N.; Medeiros, P. A.; Cordeiro, A. Chuvas intensas no Estado de Santa Catarina. Florianópolis: UFSC/EDIFURB, 2002. 156p.

Occhipinti, A. G.; Santos, P. M. Relações entre as precipitações máximas de um dia e de 24 horas na cidade de São Paulo. São Paulo: IAU/USP, 1966.10p.

Oliveira, L. F. C; Antonini, J. C.; Griebeler, N. Métodos de estimativa de precipitação máxima para o Estado de Goiás. Revista Brasileira de Engenharia Agrícola e Ambiental, v.12, p.620-625, 2008.

Pfafstetter, O. Chuvas intensas no Brasil: relação entre precipitação, duração e frequência de chuvas em 98 postos com pluviógrafos. Rio de Janeiro: DNOCS, 1957. 419p. 
Silva, D. D.; Pinto, F. R. L. P.; Pruski, F. F.; Pinto, F. A. Estimativa e espacialização dos parâmetros da equação de intensidadeduração-frequência da precipitação para os Estados do Rio de Janeiro e Espírito Santo. Revista de Engenharia Agrícola, v.18, p.11-21, 1999.

Silva, D. D.; Gomes Filho, R. R.; Pruski, F. F.; Pereira, S. B.; Novaes, L. F. Chuvas intensas no Estado da Bahia. Revista Brasileira de Engenharia Agrícola e Ambiental, v.6, p.362-367, 2002.
Soprani, M. A.; Reis, J. A. T. Proposição de equações de Intensidade-Duração-Frequência de precipitações para a bacia do rio Benevente, ES. Revista Capixaba de Ciência e Tecnologia, v.1, p.18-25, 2007.

Torrico, J. J. T. Práticas hidrológicas. Rio de Janeiro: Transcon, 1975. 119p.

Weiss, L. L. Ratio of true to fixed-interval maximum rainfall. Journal of Hydraulics Division, v.90, p.77-82, 1964. 